\title{
Multiparametric Programming in Process Systems Engineering: Recent Developments and Path Forward
}

\author{
losif Pappas ${ }^{1,2}$, Dustin Kenefake ${ }^{1,2}$, Baris Burnak ${ }^{1,2}$, Styliani Avraamidou ${ }^{2}$, Hari S. Ganesh ${ }^{2}$, \\ Justin Katz ${ }^{1,2}$, Nikolaos A. Diangelakis ${ }^{1,2}$ and Efstratios N. Pistikopoulos ${ }^{1,2 *}$ \\ ${ }^{1}$ Artie McFerrin Department of Chemical Engineering, Texas A\&M University, College Station, TX, United States, ${ }^{2}$ Texas A\&M \\ Energy Institute, Texas A\&M University, College Station, TX, United States
}

The inevitable presence of uncertain parameters in critical applications of process optimization can lead to undesirable or infeasible solutions. For this reason, optimization under parametric uncertainty was, and continues to be a core area of research within Process Systems Engineering. Multiparametric programming is a strategy that offers a holistic perspective for the solution of this class of mathematical programming problems.

OPEN ACCESS

Edited by: Mariano Martín, University of Salamanca, Spain

Reviewed by: Michael C. Georgiadis, Aristotle University of Thessaloniki, Greece

Vassilis M. Charitopoulos, University College London, United Kingdom

*Correspondence: Efstratios N. Pistikopoulos stratos@tamu.edu

Specialty section:

This article was submitted to Computational Methods in Chemical Engineering,

a section of the journal

Frontiers in Chemical Engineering

Received: 22 October 2020 Accepted: 16 December 2020 Published: 21 January 2021

Citation:

Pappas I, Kenefake D, Burnak B, Avraamidou S, Ganesh HS, Katz J, Diangelakis NA and Pistikopoulos EN (2021) Multiparametric Programming

in Process Systems Engineering:

Recent Developments and

Path Forward.

Front. Chem. Eng. 2:620168.

doi: 10.3389/fceng.2020.620168 Specifically, multiparametric programming theory enables the derivation of the optimal solution as a function of the uncertain parameters, explicitly revealing the impact of uncertainty in optimal decision-making. By taking advantage of such a relationship, new breakthroughs in the solution of challenging formulations with uncertainty have been created. Apart from that, researchers have utilized multiparametric programming techniques to solve deterministic classes of problems, by treating specific elements of the optimization program as uncertain parameters. In the past years, there has been a significant number of publications in the literature involving multiparametric programming. The present review article covers recent theoretical, algorithmic, and application developments in multiparametric programming. Additionally, several areas for potential contributions in this field are discussed, highlighting the benefits of multiparametric programming in future research efforts.

Keywords: multiparametric programming, explicit model predictive control, process systems engineering, optimization under uncertainty, data science

\section{INTRODUCTION}

What is the impact of varying parameters in mathematical optimization problems? Optimal decision-making under parametric uncertainty is a fundamental area of research within the Process Systems Engineering community since its presence in practical applications is unavoidable. Reaction kinetic constants, mass transfer coefficients, demand volumes, feedstock prices, process disturbances, appear in optimization models, and comprise sources of uncertainty in the form of parameters that can lead to inaccurate or infeasible decisions. As a result, the derivation of the optimal solution which incorporates uncertainty considerations becomes challenging.

Among the various techniques that have been proposed in the open literature to solve this problem, multiparametric programming has emerged as a powerful tool to explain how parametric uncertainty and variability influence optimal decisions. The case of single-parameter problems (i.e. parametric programming) had been investigated since the 1950s. However, the computational complexity associated with the presence of numerous parameters in the problem formulation, hindered the development of algorithms for its solution. In one of these early efforts, Barnett (1968) demonstrated 
how the range of allowable changes of parameters appearing on the optimal basis of a linear program can be calculated, along with the form of the optimal solution. Multiparametric programming attracted significant interest, most notably following the efforts of Gal and Nedoma (1972), who considered multiple parameters, and introduced a systematic strategy to solve multiparametric linear programming problems (mpLPs), and explore all optimal active sets. In their formulation, the uncertain parameters appear solely in the right-hand side of the constraints, and the solution of the aforementioned program offered an explicit relationship between the decision variables and the varying parameters. This relationship constitutes the foundation of multiparametric programming and provides a holistic view to perform uncertainty analysis in optimization problems. Hence, the optimizer solves a single mathematical programming problem to describe the impact of the uncertainty for its whole range, without the assumption of a given probability distribution. Subsequently, uncertainty in the objective function was included in the problem definition (Gal, 1975).

The seminal papers by Pistikopoulos et al. (2000) and Bemporad et al. (2002b) that demonstrated the first exact strategy for the solution of convex multiparametric quadratic programming problems (mpQPs) represent another major milestone. In addition to that, they proved that a model predictive control problem (MPC), with a quadratic cost function, can be exactly reformulated and solved as a mpQP. In this work, the optimal control actions of a system - dictated by a discrete linear time-invariant state-space model - within a MPC framework are expressed as affine functions of the initial states which partially comprise the uncertainty vector of the optimal control problem. The immediate benefits of the offline nature of the solution of the multiparametric/explicit model predictive control (mpMPC) problem are captured by the following statements

(1) The computational burden associated with solving the MPC problem is dramatically reduced since the online repetitive solution of a quadratic optimization problem is substituted by a function evaluation.

(2) The feasible state-space of the control problem is explicitly created, offering a clear picture of the operability boundaries of the system to the engineer before the start of a closed-loop simulation.

(3) The understanding of the impact of the initial states of the system to the corresponding optimal control law and the objective function value for any optimal active set.

This research breakthrough motivated a new research direction in the process control community due to the capabilities of the approach for real-time optimization problems, where the computational power is limited. Nevertheless, the aforementioned benefits of having an explicit solution come at a cost. Namely, the computational effort in deriving the full multiparametric solution depends on the number of decision variables, parameters, and constraints, and as a result, as the problem size grows, the construction of the full solution becomes challenging. Additionally, for large-scale problems the procedure of finding which optimal active set corresponds to a feasible parameter value - the point location problem - can be complex. Nowadays, research efforts could be categorized into theoretical developments to derive and store the solution of new classes of multiparametric programming problems, the construction of efficient algorithms for the exploration of the parameter space which is described by the optimal active sets, and finally, their application in engineering problems. mpMPC is by far the most well-studied and explored application of multiparametric programming. Nevertheless, this exciting field captures a generous number of applications, beyond process control. To this end, researchers adopted multiparametric programming to solve other classes of receding horizons problems, most prominently, process scheduling and moving-horizon estimation. In recent years, the area of multiparametric programming has burgeoned with advances that include its integration with data-driven modeling and optimization techniques, the interactions of process design, control, and scheduling, robust MPC, and strategies for multilevel optimization.

Several reviews have been published in the field of multiparametric programming and mpMPC (Dua et al., 2008; Alessio and Bemporad, 2009; Pistikopoulos, 2012; Oberdieck et al., 2016a). The objective of this article is to provide an update on the recent developments in theory, algorithmic strategies, and applications, which have appeared in the open literature. Additionally, the aim is to also offer a perspective on potential future research pathways which will utilize multiparametric programming-based strategies to solve challenging engineering problems, and act as a roadmap for a researcher in this field. The remainder of this work is organized as follows: In Section 2, the fundamentals of multiparametric programming are introduced, while in Section 3 recent theoretical and algorithmic contributions are presented. In Section 4 applications which have multiparametric programming in their core to solve critical engineering problems are discussed. Section 5 serves as a future outlook, and in Section 6, we conclude.

\section{BACKGROUND}

\subsection{Multiparametric Programming}

Consider a general multiparametric programming problem, described by the following formulation

$$
\begin{array}{cl}
z(\theta)=\min _{x} & f(x, \theta) \\
\text { s.t. } & g(x, \theta) \leq 0 \\
& h(x, \theta)=0 \\
& x \in \mathbb{R}^{n} \\
& \theta \in \mathbb{R}^{m}
\end{array}
$$

where $x$ is the vector of the decision variables, $\theta$ the vector of the uncertain parameters, and $g(x, \theta)$ and $h(x, \theta)$ the inequality and equality constraints respectively. Multiparametric programming is founded on the assumptions and principles of the Basic Sensitivity Theorem as presented in Fiacco (1983). Based on that, the active set in the neighborhood of a nominal parameter vector, $\theta^{*}$, with a corresponding optimal solution and Lagrange multipliers $\left(x^{\star}, \lambda^{\star}\right)$ of an optimization program, remains unchanged. Consequently, parametric areas with the same active set are created - critical regions - each characterized with a distinct set of Karush-KuhnTucker (KKT) conditions. Since the optimality conditions remain the same, the resulting system of equations derived from the KKT conditions can be analytically solved, and hence the optimal solution 
with respect to the varying parameters for the whole parameter space is explicitly constructed. This statement can be expressed as

$$
x^{*}(\theta)=\left\{\begin{array}{ccc}
\mathrm{x}^{1}(\theta), & \text { if } & \theta \in \mathrm{CR}^{1} \\
\mathrm{x}^{2}(\theta), & \text { if } & \theta \in \mathrm{CR}^{2} \\
\cdot & & \\
\cdot & & \\
\cdot & & \\
\mathrm{x}^{\mathrm{v}}(\theta), & \text { if } & \theta \in \mathrm{CR}^{\mathrm{v}}
\end{array}\right.
$$

where the $i^{\text {th }}$ critical region, $C R^{i}$, corresponds to the parameter space described by the $i^{\text {th }}$ active set. Consequently, the full solution of this optimization problem is obtained beforehand, and by identifying a given parameter value, the optimal solution is reduced to a simple function evaluation. The procedure of finding in which region a measured value of parameters lies, is termed as the point location problem. A schematic of a map of critical regions is shown in Figure 1.

Depending on the structure of problem 1, the functions describing the multiparametric solution and the structure of the critical regions can drastically change. General closed-forms for the optimal solutions are known for mpLPs, mpQPs, and their mixedinteger counterparts (mpMILPs, mpMIQPs). Specifically, assume the following mpMIQP that includes both continuous optimization variables, $x$, and binary variables, $y$

$$
\begin{aligned}
z(\theta)=\min _{x, y} & (Q \omega+H \theta+c)^{T} \omega \\
\text { s.t } & W_{i} x+E_{i} y \leq b_{i}+F_{i} \theta \\
& W_{e} x+E_{e} y=b_{e}+F_{e} \theta \\
& x \in \mathbb{R}^{n}, y \in\{0,1\}^{p}, \omega=\left[x^{T} y^{T}\right]^{T} \\
& \theta \in \mathbb{R}^{m}
\end{aligned}
$$

where the problem is defined by the matrices of appropriate dimensions.

Table 1 demonstrates the properties of the multiparametric solution depending on the problem type. Please note that problem 3 includes all the aforementioned problem formulations.

\subsection{Connecting Multiparametric Programming and Model Predictive Control}

A model predictive control problem, whose goal is to drive the system to the origin, can be described by the following formulation, assuming that $\hat{x}_{k}$ is the vector of the states of the system, $\widehat{y}_{k}$ the outputs, and $\widehat{u}_{k}$ are the control actions to be found at time instant $k$

$$
\begin{array}{lr}
\min _{\widehat{u}_{0}, \ldots, \widehat{u}_{N-1}} \widehat{x}_{N}^{T} P \widehat{x}_{N}+\sum_{k=0}^{N-1} \widehat{x}_{k}^{T} Q_{R} \widehat{x}_{k}+\widehat{u}_{k}^{T} R \widehat{u}_{k} \\
\text { s.t. } \widehat{x}_{k+1}=A \widehat{x}_{k}+B \widehat{u}_{k} & k=0, \ldots, N \\
\widehat{y}_{k}=C \widehat{x}_{k} & k=0, \ldots, N \\
\widehat{x}_{k} \in \mathbb{X} & k=0, \ldots, N \\
\widehat{u}_{k} \in \mathbb{U} & k=0, \ldots, N-1 \\
\widehat{x}_{0}=\widehat{x}(0) & \\
\widehat{x}_{N} \in \mathbb{T} &
\end{array}
$$

where $Q_{R}$ and $R$ are the weights of the objective function, and $P$ is the stabilizing matrix derived by the solution of the Riccati equation for discrete systems, and $N$ is the prediction horizon of the problem. It is assumed that the sets $\mathbb{X}, \mathbb{U}$, and $\mathbb{T}$ are compact, the matrices $Q_{R}$ and $P$ are positive semi-definite, while $R$ is positive definite. Problem 4 is dictated by a discrete linear statespace model. Hence, future state vectors can be expressed as a function of the initial state of the system and the control actions, by utilizing the following relationship

$$
\widehat{x}_{k}=A^{k} \widehat{x}_{0}+\sum_{q=0}^{k-1} A^{q} B \widehat{u}_{k-1-q}
$$

Assuming that the initial state vector $\widehat{x}_{0}$ is a vector of uncertain parameters, problem 4 can be reformulated to 3 , and the optimal control policy can be calculated as an affine function of this uncertainty vector. Through mpMPC additive disturbances, setpoints, and outputs can also be part of the problem formulation.

\section{THEORETICAL AND ALGORITHMIC DEVELOPMENTS}

The benefits of multiparametric programming-based solutions in practical applications have created a dynamic interest by the research community over the years, and resulted in multiple theoretical and algorithmic developments. Specifically, these contributions were focused on 1) the solution of new classes of multiparametric programming problems, and 2) the derivation of algorithms for the exploration of the parameter space.

\subsection{Multiparametric Nonlinear Programming}

The need to tackle more complex problems, described by nonlinear functions, is a central area of research within the multiparametric programming community. Even though linear models are shown to perform well in a wide range of optimization applications, such as model predictive control, various processes are inherently nonlinear. Apart from that, the required fluctuations in operation, together with stricter environmental and financial targets, lead to the need of incorporating nonlinear terms in these optimization formulations. Researchers focused on the impact of a single parameter to calculate how that would affect the behavior of a nominal solution of general nonlinear programs (Kojima, 1979; Benson, 1982; Kojima and Hirabayashi, 1984). The foundations of modern algorithms in nonlinear multiparametric programming have been based on the results of Fiacco and their coworkers, who provided local regularity and local sensitivity conditions for the general case involving multiple parameters (Fiacco, 1976; Fiacco, 1983; Fiacco and Kyparisis, 1986). More recent efforts in multiparametric nonlinear programming problems (mpNLPs) concentrated on developing approximations of the mpNLP to a form that exact solution strategies exist, by creating linear or quadratic approximations of the objective function and linearization of the constraints (Dua 
and Pistikopoulos, 1999; Johansen, 2002; Domínguez and Pistikopoulos, 2013). Apart from that geometric-based strategies have been utilized by creating parameter space approximation through hypercubes, simplicies, and hyperrectangles (Johansen, 2004; Bemporad and Filippi, 2006; Narciso, 2009), the utilization of a partially offline solution (Fotiou et al., 2006), or the approximation of the solution manifold based on the Fritz John conditions of optimality (Hale and Qin, 2004; Hale, 2005).

Dua (2015) considered general multiparametric mixed-integer nonlinear programming problems (mpMINLPs) described by polynomial functions. The author derived and solved the system of polynomial equations resulting from the KKT conditions, where the integer variables are treated as uncertain parameters. Since the problems include integer and nonconvex functions, the solutions of the KKT system are checked to identify whether the inactive inequality constraints, the slack complementary slackness and the constraint qualifications are satisfied. In Charitopoulos and Dua (2016) hybrid nonlinear model predictive control problems were considered, and the problem is reformulated as a mixed-integer polynomial programming problem. The algorithmic approach is based on the analytical solution of the system of equations arising from the KKT conditions. After performing feasibility and constraint qualification investigations of the solutions, a dominance check is performed for overlapping critical regions, leading to an exact multiparametric solution.

In another contribution, the focus was on controlling spatially distributed systems described by partial differential equations (PDEs) (Petsagkourakis and Theodoropoulos, 2018). A reducedorder digital twin of the process was constructed using proper orthogonal decomposition followed by a neural network. Given the challenges of directly solving the resulting mpNLP, multiple linearizations of the nonlinear problem were created, resulting in mpQPs. A model approximation checking mechanism was also employed that verifies whether the produced approximation error bound was satisfied, or further refinement of the model was required. A different perspective in solving mpNLPs in the form of mpMPC was given in Bayer et al. (2016). The authors created a grid of the state-space and assume a control law parametrization. A suboptimal control law, valid for a set of initial conditions, is computed through robust tube-based MPC techniques (Langson et al., 2004). As a result, hyperrectangular regions are created. However, the proposed approach has also an online cost, associated with finding the region with the desired control law.

In contrast to other efforts which consider general mpNLPs, in Diangelakis et al. (2018); Pappas et al. (2020b) the authors restricted themselves to multiparametric quadratically constrained quadratic programming problems (mpQCQPs), motivated by the applications described by this class of problems. However, the given structure of the problem allowed them to systematically show how the explicit solution for this class of problems can be generated without a postprocessing step. The approach is based on a second-order Taylor approximation of the Basic Sensitivity Theorem, which allows for the existence and derivation of the multiparametric solution. In addition to the case where only convex constraints are included in the problem description, the authors expanded to the case where nonconvex constraints are part of the optimization formulation. The incorporation of quadratic terms in the constraints results in the construction of nonlinear and nonconvex critical regions. Hence, the adoption of a geometric strategy is challenging due to the absence of facets to utilize to explore new critical regions. For this reason, the authors utilize an active set-based exploration algorithm to guarantee the complete characterization of the parameter space. A crucial point in this procedure is also the fact that the comparison of overlapping critical regions is avoided, since the resulting Lagrange function is guaranteed to be convex. This development has been utilized for the solution of quadratically constrained model predictive control problems and the flexibility index problems with quadratic constraints and design considerations (Pappas et al., 2020a; Pappas et al., 2020b).

Based on the presented solution strategies for mpNLPs, there are two main routes that this class of problems has been approached. Firstly, an approximate solution, founded on a mpLP or mpQP, can be derived which has advantages in solving larger-scale problems. However, an approximation step will potentially encompass inaccuracies that can lead to undesirable behavior or infeasibility when applied to an actual problem. On the other hand, exact solution approaches result into an accurate solution. The fundamental challenge with exactly dealing with mpNLPs is the required analytical solution of the resulting system of nonlinear equations derived from the KKT conditions, which will express the decision variables as a function of the uncertain parameters. In Dua (2015); Charitopoulos and Dua (2016); Pappas et al. (2020b), Gröbner bases have been utilized - which scale with the number of decision variables - to build the parametric solution. However, among the computational algebra techniques, they have shown to be effective in solving nonlinear equations analytically, and further developments in parallel computing will encourage their wider adoption in multiparametric programming.

\subsection{Parameter Space Exploration}

A critical point in the multiparametric programming solution construction process is the efficiency of exploring the parameter space. Strategies to achieve that still remains an active area of research. Initially, approaches that solely observe the parameter space had been suggested. Namely, assuming that a single critical region is discovered, these exploration algorithms identify geometrically adjacent critical regions. The procedure is repeated until all regions are found. On the other hand, active set-based strategies exploit the fact that the optimal solution is defined by the active constraints, and hence by considering every active set in the parameter space, all critical regions are found. As the former techniques scale unfavorably with the number of parameters of the problem, and the latter with the number of decision variables and constraints, the selection of the exploration algorithm depends on the structure of the optimization problem. Moreover, note that that geometric-based techniques do not guarantee the full parameter space exploration. In the past three years, hybrid algorithms that blend the benefits of both 


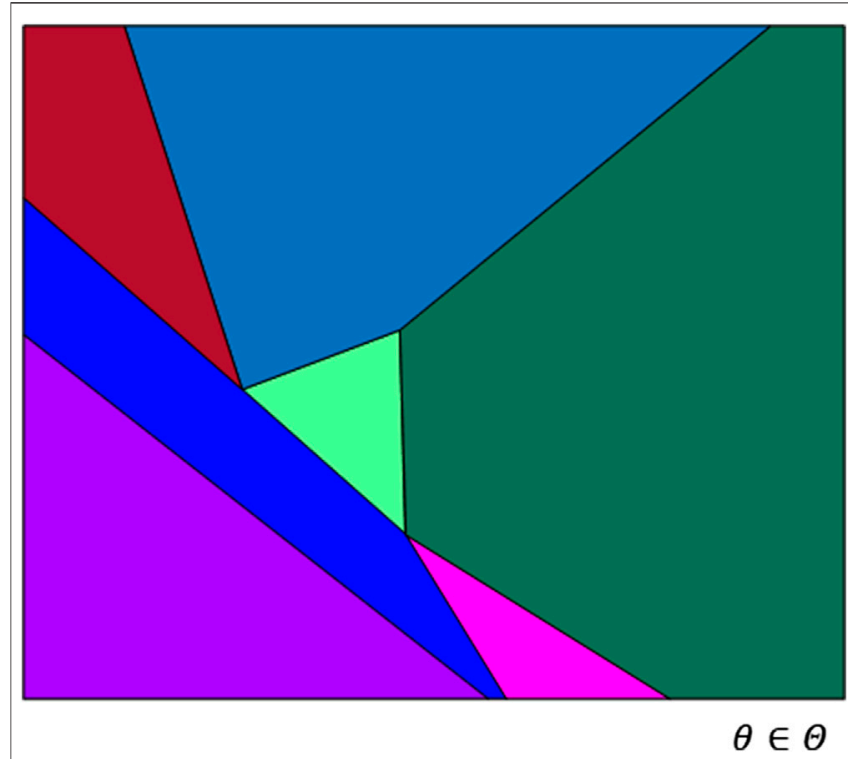

Figure 1 | Schematic representation of critical regions resulting from the solution of a multiparametric programming problem. Each region is described by a different optimal active set.

approaches have been published, and have been extensively used in various applications. In Table 2 the algorithms that can solve the classes of problems that have general closed-form solutions are presented.

Ahmadi-Moshkenani et al. (2018) proposed a hybrid multiparametric exploration approach for mpQPs, in a similar spirit to the one proposed by Oberdieck et al. (2017b). In particular, the devised algorithm is an active set-based in its core, and as a result, assumes a candidate active set to be potentially optimal. Subsequently, it asks the question whether there is a parameter vector value that the optimality assumption holds true. Additionally, to reduce the computational complexity, the authors take advantage of the facet-to-facet property, which states that two adjacent critical regions differ by a single constraint in their active set. Hence, for an already explored critical region, they add or remove a constraint in the construction of the optimality conditions and verify if that holds.

In Akbari and Barton (2018), multiparametric programming was studied through the lenses of metabolic networks and flux balance analysis. A key focus of this contribution is primal degeneracy and multiplicity of mpLPs. By exploiting the structure of the studied problems, the authors show that the issue of having an overdetermined KKT system with respect to the decision variables can be solved through generalized inverses, with enhanced computational complexity to the already published approaches. Moreover, the case where there are multiple primal solutions was addressed through an auxiliary objective or by solving a lexicographic linear program. An extensive discussion in degeneracy in multiparametric programming, and ways to tackle it is also presented in Oberdieck et al. (2016a).

Burnak et al. (2020b) proposed a space exploration algorithm for mpLPs, mpQPs, and mpMILPs. Their approach is facilitated by constructing simplices of the parameter space through Delaunay triangulation. Deterministic optimization problems are solved at the vertices of these simplices based on which parameter areas described by the same optimality conditions are identified. As a result, optimal parameter space partitions are created. Furthermore, it is shown that through the proposed algorithm volumetrically significant critical regions are prioritized to be discovered.

\section{APPLICATIONS}

Multiparametric programming theory has been utilized to tackle various challenging problems. In this section, application areas that have utilized multiparametric programming at their heart for their solution are presented.

\subsection{Multiparametric/Explicit Model Predictive Control}

Multiparametric/explicit model predictive control is the most well-studied application of multiparametric programming. Since its inception (Pistikopoulos et al., 2000; Bemporad et al., 2002b), countless implementations and variations of the approach have been published in the literature both in simulations and in practical applications, which contribute to the thousands of citations of the original papers. Extensive reviews in mpMPC have been published in the literature to capture the use of multiparametric programming in process control (Dua et al., 2008; Alessio and Bemporad, 2009; Pistikopoulos et al., 2015). It is to be highlighted that large-scale industrial applications have utilized and will continue to utilize an online optimization algorithm for MPC. These implementations are used to control slow processes such as oil refineries and chemical manufacturing plants, which have already invested in installing a form of online MPC (Qin and Badgwell, 2000; Qin

Table 1 | Properties of multiparametric programming problems that have general closed-form solutions.

\begin{tabular}{|c|c|c|c|}
\hline Problem type & $\begin{array}{l}\text { Multiparametric solution, } \\
\qquad \boldsymbol{x}(\theta)\end{array}$ & Objective function, $\boldsymbol{z}(\theta)$ & Critical regions, $C \boldsymbol{R}_{\boldsymbol{i}}$ \\
\hline mpLP & Piecewise affine & Piecewise affine & Polytopic \\
\hline mpMILP & Piecewise affine & Piecewise affine & Polytopic/Nonlinear \\
\hline $\mathrm{mpQP}$ & Piecewise affine & Piecewise quadratic & Polytopic \\
\hline mpMIQP & Piecewise affine & Piecewise quadratic & Polytopic/Nonlinear \\
\hline
\end{tabular}


Table 2 | Multiparametric programming algorithms for the solution of mpLPs, mpMPs, mpMILPs, and mpMIQPs.

\begin{tabular}{|c|c|c|c|c|}
\hline & mpLP & mpMILP & mpQP & mpMIQP \\
\hline Gal and Nedoma (1972) & $x$ & & & \\
\hline Gal (1975) & $x$ & & & \\
\hline Yuf and Zeleny (1976) & $x$ & & & \\
\hline Schechter (1987) & $x$ & & & \\
\hline Acevedo and Pistikopoulos (1997) & $x$ & $x$ & & \\
\hline Pertsinidis et al. (1998) & $x$ & $x$ & & \\
\hline Pistikopoulos et al. (2000) & $x$ & & $x$ & \\
\hline Dua and Pistikopoulos (2000) & $x$ & $x$ & & \\
\hline Bemporad et al. (2002b) & $x$ & & $x$ & \\
\hline Dua et al. (2002) & $x$ & $x$ & $x$ & $x$ \\
\hline Bemporad et al. (2002a) & $x$ & & & \\
\hline Baotic (2002) & $x$ & & $x$ & \\
\hline Tøndel et al. (2003) & $x$ & & $x$ & \\
\hline Filippi (2004) & $x$ & & & \\
\hline Jia and lerapetritou (2006) & $x$ & $x$ & & \\
\hline Spjøtvold et al. (2006) & $x$ & & $x$ & \\
\hline Jones et al. (2007) & $x$ & & & \\
\hline Li and lerapetritou (2007) & $x$ & $x$ & & \\
\hline Faísca et al. (2009) & $x$ & $x$ & & \\
\hline Mitsos and Barton (2009) & $x$ & $x$ & & \\
\hline Patrinos and Sarimveis (2010) & $x$ & & $x$ & \\
\hline Li and lerapetritou (2010) & $x$ & $x$ & $x$ & $x$ \\
\hline Gupta et al. (2011) & $x$ & & $x$ & \\
\hline Feller et al. (2013) & $x$ & & $x$ & \\
\hline $\begin{array}{l}\text { Wittmann-Hohlbein and Pistikopoulos } \\
\text { (2013) }\end{array}$ & $x$ & $x$ & & \\
\hline Oberdieck et al. (2014) & $x$ & $x$ & & \\
\hline $\begin{array}{l}\text { Wittmann-Hohlbein and Pistikopoulos } \\
\text { (2014) }\end{array}$ & $x$ & $x$ & & \\
\hline Axehill et al. (2014) & $x$ & $x$ & $x$ & $x$ \\
\hline Bemporad (2015) & $x$ & & $x$ & \\
\hline Herceg et al. (2015) & $x$ & $x$ & $x$ & $x$ \\
\hline Oberdieck and Pistikopoulos (2015) & $x$ & $x$ & $x$ & $x$ \\
\hline Charitopoulos et al. (2017) & $x$ & & & \\
\hline Oberdieck et al. (2017b) & $x$ & & $x$ & \\
\hline Charitopoulos et al. (2018) & $x$ & $x$ & & \\
\hline Ahmadi-Moshkenani et al. (2018) & $x$ & & $x$ & \\
\hline Akbari and Barton (2018) & $x$ & & & \\
\hline Burnak et al. (2020b) & $x$ & $x$ & $x$ & \\
\hline
\end{tabular}

and Badgwell, 2003). Also, the scale of the problem for such implementations makes the derivation of the full multiparametric solution substantially challenging, given that they are comprised by a significant number of variables, states, and constraints. Nevertheless, the benefits of mpMPC are amplified in small to medium-scale applications. In these cases, there might exist some control hardware and software infrastructure to achieve the control objective, but due to lack of computational power or other constraints (e.g. power requirements, cost), the use of online MPC is not suitable. For such processes, whose dynamics are typically fast, mpMPC (or MPC-on-a-chip) can be an advantageous choice (Alessio and Bemporad, 2009; Pistikopoulos, 2009). Hence, numerous applications of mpMPC on simulation and real-life laboratory and pilot problems have been recorded in the published literature. The latter include biomedical systems (Dua, 2005), separation units (Grancharova et al., 2004; Mandler et al., 2006), automotive systems (Di Cairano et al., 2008; Naus et al., 2008), power electronics (Linder and Kennel, 2005; Mariéthoz and Morari, 2008), and energy systems (Arce et al., 2011). Accounting to its potential and recent advancements, $\mathrm{mPMPC}$ has continued to be applied in several fields and realistic problems, that are highlighted on Table 3.

It is expected that mpMPC, similarly to the online MPC, will continue to find success and applicability in various fields (Mayne, 2014). Particular emphasis will be paid on areas that do not have the capability of having a powerful computer machine to perform online calculations to solve the optimal control problem.

\subsection{Design, Control, and Scheduling}

The Process Systems Engineering community has considered the seamless integration of process design and operational decisions a vital direction to achieve improved process efficiency and reliability (Burnak et al., 2019b). Conventional techniques for process decisions are usually isolated from each other with a hierarchical order in the time scales they span. Such isolation yields suboptimal, even infeasible operations when the decisions are applied in real time, due to lack of perfect information flow across different decision layers (Mohideen et al., 1996; Pistikopoulos and Diangelakis, 2016; Caspari et al., 2020). The integrated problem is mathematically casted as a multilevel dynamic optimization problem, hence conventional global optimization techniques are unable to solve this class of problems without simplifying assumptions or approximations. Furthermore, the time scale differences between each level exacerbate the tractability of the integrated problem (Rafiei and Ricardez-Sandoval, 2020). Multiparametric programming has been shown to be an effective tool to develop a simultaneous framework for here-and-now design decisions and operational decisions including process control, scheduling, and planning. The explicit solution set, described by Eq. 2, provides an offline map of optimal operational decisions that can be integrated in a dynamic optimization problem that governs longer time horizons. Therefore, the faster time scale decisions can be formulated as a multiparametric programming problem, where the unrealized system parameters at their respective levels can be explicitly accounted for, and incorporated into an overarching optimization problem with slower process dynamics (Burnak et al., 2020a).

Sakizlis et al. (2003) presented one of the first significant attempts to use multiparametric programming for the integration of process design and operational optimization problems. The authors integrated the explicit solution of an mpMPC into a mixed integer dynamic optimization problem that simultaneously minimizes the capital and operating costs. Although the approach proposed by Sakizlis et al. (2003) was shown to be effective as showcased on a binary distillation column and an evaporator, it relied on repetitive linearizations and solving a multiparametric programming problem at every iteration. Diangelakis et al. (2017) improved this approach by deriving a "design dependent offline controller", which accepted the design variable as a parameter in the mpMPC problem, allowing for the formulation of a single mixed integer dynamic optimization formulation to determine the optimal 
Table 3 | List of recent real-life laboratory and pilot-scale applications of multiparametric/explicit model predictive control.

\begin{tabular}{|c|c|c|}
\hline Area & Contribution & Description \\
\hline \multirow[t]{6}{*}{ Automotive } & Emekli and Güvenç (2016) & Pressure control of a diesel engine \\
\hline & Vadamalu and Beidl (2016) & Energy management of hybrid vehicles \\
\hline & Theunissen et al. (2019) & Suspension systems with road prediction \\
\hline & Tavernini et al. (2018) & Wheel control of an electric vehicle \\
\hline & Tavernini et al. (2019) & Antilock breaking system for breaking \\
\hline & Lee and Chang (2019) & Autonomous steering control \\
\hline \multirow[t]{5}{*}{ Energy } & Lasheen et al. (2019) & Pitch angle control in wind turbines \\
\hline & Ogumerem and Pistikopoulos (2019) & Temperature control for metal-hydrides \\
\hline & Ogumerem and Pistikopoulos (2020) & Water electrolysis for hydrogen production \\
\hline & Drgoňa et al. (2017) & Water/methanol distillation column \\
\hline & Ziogou et al. (2018) & Polymer electrolyte membrane fuel cell \\
\hline \multirow[t]{2}{*}{ Power electronics } & Klaučo et al. (2017) & Magnetic levitation control \\
\hline & Jia et al. (2019) & Current control of a synchronous motor \\
\hline Biomedical & Scaglioni et al. (2019) & Control of a flexible magnetic endoscope \\
\hline Robotics & Ettefagh et al. (2019) & Vibration control of flexible joints \\
\hline
\end{tabular}

design and closed loop control strategies. Tian et al. (2020) showed that a similar systematic approach is also applicable for much more complex intensified systems, namely a reactive distillation process.

On the integration of the operational decisions, Zhuge and Ierapetritou (2014) integrated the explicit MPC expressions in a continuous time, event point based scheduling problem for a batch process. Burnak et al. (2018) derived the offline expressions both for the scheduling and the MPC problems, and addressed the time scale gap by developing offline scale bridging models. Charitopoulos et al. (2019) incorporated nonlinear mpMPC, and extended the problem to include the planning problem.

A complete theory and framework for the integration of process design, scheduling, and control decisions was recently proposed by Burnak et al. (2019a), where the authors derived design dependent offline control and scheduling strategies that are embedded exactly in a mixed integer dynamic design optimization problem via multiparametric programming. Burnak and Pistikopoulos (2020) extended this framework to batch processes, where the scheduling of the multipurpose batch units is represented by a State Equipment Network (SEN).

\subsection{Multilevel Optimization}

Optimization problems involving a set of nested optimization problems over a single feasible region are referred to as multilevel programming problems. The control over the decision variables is divided among different optimization levels, but all decision variables can affect the objective function and constraints of all optimization levels. This class of problems has attracted considerable attention across a broad range of research communities, including economics, sciences, and engineering, and has been applied to many diverse problems that require hierarchical decision making.

Since the early 1980s, many algorithms have been proposed for the solution of continuous bilevel problems with many approaches exploiting the KKT optimality conditions of the lower level problem, to transform the multilevel problem into a single level problem. The main idea behind the development of multiparametric algorithms for the solution of multilevel programming problems came out through the observation that in a multilevel optimization setting, the lower level optimization problems are parametric in terms of the upper level variables (Dempe, 2002). This observation gave rise to the development of several algorithms for the solution of multilevel problems with the key idea to solve the lower level problem parametrically in terms of the upper level variables and transform the multilevel problem into a set of single-level reformulations.

Bilevel programming has attracted the most attention amongst other classes of multilevel programming problems due to its simplicity (compared to other multilevel problems) and great applicability. Multiparametric programming-based approaches for the solution of linear and quadratic bilevel problems were firstly introduced (Ryu et al., 2004; Faísca et al., 2007; FaÃsca et al., 2011). For classes of problems where the lower level problems also involve discrete variables, multiparametric solution algorithms can alleviate the need for global optimization methods and offer the exact global solution to bilevel mixed-integer linear and quadratic optimization problems (Domínguez and Pistikopoulos, 2010; Oberdieck et al., 2017a; Avraamidou and Pistikopoulos, 2019d).

The solution of optimization problems with more than two optimization levels has been addressed only for a restricted class of problems, mainly continuous trilevel linear problems, with only a few attempts to solve problems with more than three optimization levels or integer variables. The developed algorithms mainly fall in two categories: 1) data driven approaches (Pramanik and Roy, 2007; Sakawa and Matsui, 2014) that cannot guarantee neither feasibility nor optimality, and 2) multiparametric based approaches (Faísca et al., 2009; Kassa and Kassa, 2013; Kassa and Kassa, 2016; Avraamidou and Pistikopoulos, 2019b; Avraamidou and Pistikopoulos, 2019c) that can find the exact global optimum for mixedinteger linear and quadratic optimization problems.

The extension to multilevel problems with multiple followers has not received a lot of attention from the research community with some attempts to solve the linear and quadratic continuous case (Faísca et al., 2009), and limited heuristic approaches for the 
solution of nonlinear mixed-integer multifollower problems (Kassa and Kassa, 2017; Avraamidou and Pistikopoulos, 2018).

Most recently, Avraamidou and Pistikopoulos (2019a) have developed a freely accessible toolbox for the solution of bilevel and trilevel, continuous and mixed-integer, linear and quadratic optimization problems through multiparametric programming, and presented applications in hierarchical model predictive control (Avraamidou and Pistikopoulos, 2017a), supply chain planning (Avraamidou and Pistikopoulos, 2017b) and planning and scheduling integration (Avraamidou and Pistikopoulos, 2018).

\subsection{Integration of Machine Learning and Multiparametric Programming}

Apart from the aforedescribed developments, the explosion of interest in state-of-the-art data science strategies has been translated into the multiparametric programming community, making it arguably one of the most active research areas in the field. One major category of efforts are focused on approximating an MPC policy through machine learning techniques (Parisini and Zoppoli, 1995; Åkesson and Toivonen, 2006). It is crucial to note that in these types of methodologies, even though an explicit form of the control law can be created such as through a neural network, they do not capture all benefits of mpMPC, such as the development of a map of solutions. In Shokry et al. (2016), Shokry et al. (2017), the authors utilized sampling strategies to pass various parameter values to the optimizer whose output is collected. Subsequently, machine learning algorithms are used to learn optimal decisions as a function of the varying parameters. Another strategy to use data-driven techniques to approximate the explicit optimal control law of a MPC problem is presented in Chen et al. (2018). Deep reinforcement learning is applied to train a deep neural network with rectified linear units (ReLU) which is used to approximate the optimal piecewise control law. Karg and Lucia (2020) utilized ReLU-based neural networks to represent the piecewise affine law of a linearly constrained mpMPC. Lovelett et al. (2020) presented a framework to learn the optimal control policy as a function of the system by using diffusion maps. The authors demonstrate that the proposed approach can be used to control both linear and nonlinear models.

The impact of machine learning models to approximate nonlinear functions for modeling purposes in the context of mpMPC has also been explored. Most notably, Katz et al. (2020b) presented a framework for the integration of deep neural networks with ReLU as activation functions and multiparametric programming. Given that a ReLU-based neural network includes max operators which are challenging to be introduced in multiparametric optimization formulation, the authors reformulated the neural network as a mixed-integer linear model that can be readily solved through existing multiparametric programming algorithms. Because the neural network to the mixedinteger linear reformulation is exact, no information is lost in the approximation step (Grimstad and Andersson, 2019), which assists in achieving a high-quality optimal multiparametric solution. The authors also applied the approach to a solar field (Katz et al., 2020a).
Apart from that, multiparametric programming has been coupled with machine learning techniques for process monitoring, in a contribution by Onel et al. (2019). In particular, support vector machine feature-based selection and modeling is used to identify potential faults that exist in a given process. This information is then processed by a random forest algorithm that calculates the magnitude of the present fault. Additionally, these faults are treated in the mpMPC design stage as uncertain parameters, and consequently, the controller acts accordingly based on the existence/absence and the magnitude of a process fault, resulting to an enhanced closedloop performance of the system.

Finally, multiparametric programming has found application as a means to enhance the capabilities of machine learning itself. A connection between hyperparameter optimization strategies, where the aim is to optimally find the algorithm parameters that control how a machine learning model learns from data, and parametric optimization has been established. Specifically, it has been shown that there is a piecewise linear parametric relationship of the model weights and the regularization term in LASSO regression (Efron et al., 2004) and support vector machine (SVM) classification (Hastie et al., 2004). Extensions for the case of multiple parameters in hyperparameter optimization problems have subsequently been presented in Karasuyama et al. (2012); Zhou and Spanos (2016). In a more recent effort, Tso et al. (2020) demonstrated that K-fold cross-validation in hyperparameter optimization can be cast as a bilevel optimization program, and exactly solved in a single formulation through multiparametric programming (Avraamidou and Pistikopoulos, 2019a). One of the key benefits of the aforementioned technique is that it can be applied to any machine learning problem that is described by a LP/QP/MILP/MIQP model.

Machine learning and data science techniques will continue to be a major part of future developments in the field of multiparametric programming. Data-driven techniques can provide assistance in solving mpNLPs, reducing the complexity from a derived multiparametric solution, solving large-scale problems, and many more.

\section{FUTURE OUTLOOK}

\subsection{Software}

Rapid progress in computational hardware has led to a widespread adoption of parallel programming and heterogeneous compute in the last 2 decades (Fang et al., 2020). Multi-core and many-core compute paradigms will be essential for performance improvements to be realized for new software. Multiparametric solvers will need to be written so that they are able to scale with hardware. Recently there has been activity in the use of parallel techniques in a multiparametric programming context (Oberdieck and Pistikopoulos, 2016; Jiang et al., 2020), however these are the solitary examples that integrate multiparametric programming and parallel computing in the open literature. There is still a large amount of work to be done in developing parallel algorithms to utilize multicore 
CPUs, as well as algorithms that distribute work across multiple compute nodes. Currently, the literature on Field Programmable Gate Arrays (FPGA) MPC is based on solving the optimization problem online (McInerney et al., 2018). Software support of heterogeneous compute platforms such as FPGA and Application Specific Integrated Circuits (ASIC) for accelerating the solution of the point location problem has an opportunity to surpass the fastest MPC implementations by exchanging the optimization problem with the point location problem.

The major multiparametric solvers, POP and MPT3 (Oberdieck et al., 2016b; Herceg et al., 2013), are both written in MATLAB. This presents challenges with inter-operability of leading software packages in data science. Implementing code generation techniques that allow the multiparametric solver to export solutions to different code environments for example $\mathrm{C}$ code for microcontrollers, Javascript code for scripting interfaces, or Python for software interfacing. The MPT3 solver implements $\mathrm{C}$ and Python generation (Takács et al., 2016). This is an important feature as it allows for the deployment of multiparametric solutions to multiple different hardware and software platforms.

Region-free multiparametric programming solutions are based on storing the active set combinations defining solution and not constructing the explicit critical regions. Instead of a more typical point location algorithm, a direct enumeration of the primal and dual constraints of each active set is carried out, as opposed to checking the reduced primal constraints in typical point location problem (Kvasnica et al., 2015). This approach makes a trade off between memory required to store the solution and the computational requirement to solve the region-free online point location problem. Methods like this could be effective for multiparametric programming on memory constrained systems.

\subsection{Complexity Reduction}

The complexity in deriving the full multiparametric programming solution is non-polynomial in nature, due to the possible combinatorial number of critical regions that define the solution of multiparametric programming problems. However, for many multiparametric programming problems the number of fully-dimensional critical regions usually not scale combinatorially, such as in generating mpMPCs. Recent algorithmic developments are based on reducing the number of deterministic optimization subproblems that need to be considered (Gupta et al., 2011). The graph-based algorithms of (Oberdieck et al., 2016a) and (Ahmadi-Moshkenani et al., 2018) greatly reduce the required number of subproblems, however they both have cases where a combinatorial number of subproblems would need to be considered for the construction of a single critical region. The development of a multiparametric programming algorithm that is output sensitive to the number of solution critical regions without the aforementioned behavior would be a significant development for the field. For specific multiparametric programming problem types, there are possibly ways to exploit the problem structure to reduce the number of deterministic optimization subproblems required to fully solve the problem, in a similar manner to the exploitation of symmetries in the constraint matrix in (Feller et al., 2013). Additionally, the online cost associated with multiparametric methods is primarily derived from solving the point location problem. There are promising results that reduce the algorithmic complexity of this operation by using bounding volume hierarchies (Tøndel and Johansen, 2002), or hash functions (Bayat et al., 2010). Further exploration of different hierarchical data structures or mathematical reformulations of the point location problem could further these results. Results from the universal approximation theorem show that any piecewise affine function can be represented by a ReLU neural network of sufficient depth (Hanin and Sellke, 2018). Advances in machine learning has enabled the construction of ReLU-based well fitting approximations of multiparametric programming solutions (Chen et al., 2018; Karg and Lucia, 2020), that will continue to be part of future developments.

\subsection{Robust Optimization and Robust Model Predictive Control}

Multiparametric programming theory has assisted in developing solution algorithms for other classes of optimization problems under uncertainty. Classical robust optimization (RO) assumes that all decisions must be made before the realization of uncertainty, a strategy that may be overly conservative, as it aims to immunize against any uncertainty. A less conservative approach is adjustable robust optimization (ARO) which involves recourse decisions (i.e. reactive actions after the realization of the uncertainty) as functions of the uncertainty (Ben-Tal et al., 2004). Solving ARO problems is challenging, therefore ways to reduce the computational effort have been proposed, with the most popular being the affine decision rules, where the recourse decisions are approximated as affine adjustments of the uncertainty (Kuhn et al., 2011); an approximation which can be overly conservative for general ARO problems. Multiparametric programming has been used to develop the exact and optimum generalized piecewise affine decision rule for mixed-integer linear ARO problems with a decision dependant uncertainty set (Avraamidou and Pistikopoulos, 2020). This offered a theoretical justification - via multiparametric programming - on why affine rules can be suboptimal (or even optimal at limited cases).

Applications of RO techniques have also been used in mpMPC. Model predictive control, being a model-based control technique, requires an accurate process to drive a given system to the desired state. Nevertheless, the underlying model in the MPC application includes uncertainty in its dynamics, since it will never be an exact representation of the real process Mohideen et al. (1997). For this reason, robustness considerations have been introduced in mpMPC studies to address this issue, as a means to hedge against model uncertainty (Kothare et al., 1996; Bemporad and Morari, 1999; Mayne et al., 2005). The field of robust MPC through multiparametric programming has not seen extensive developments due to the inherent computational complexity stemming from the need to obtain feasible operation. Sakizlis et al. (2004) included a feasibility constraint in the mpMPC 
formulation assuming linear models with additive uncertainty, and $1 / \infty$ or quadratic performance indices. Tejeda-Iglesias et al. (2019) utilized ARO to develop a mpMPC policy for discrete linear state-space plants with additive disturbances. On the other hand, Bemporad et al. (2003) considered linear uncertain systems (problems with multiplicative uncertainty) with a linear objective function. Kouramas et al. (2013) proposed a solution for uncertain linear systems with a quadratic performance criterion, using dynamic programming, which required the solution of a multiparametric programming problem at each stage of the prediction horizon. The solution of multiple multiparametric programs poses a significant challenge in the derivation of the solution. Hence, an open question in this area is the development of an approach for robust mpMPC problems with a quadratic objective function avoiding the need of dynamic programming. Furthermore, since the robust control formulation considers a larger number of uncertainty sources compared to the conventional MPC, solution techniques that would be able to efficiently manage the complexity stemming from the increased parameter space dimensionality is needed.

\subsection{Multiparametric Programming for Online Optimization}

A significant number of researchers have solved multiparametric programming problems without developing the full map of solutions. Instead, the utilized concepts of multiparametric programming theory to shrink the online cost associated with the solution of the original problem. This strategy has been preferred in large scale online applications, where the derivation of the full multiparametric solution is prohibitive.

An approach that utilizes concepts of multiparametric programming for large-scale optimization applications is shown in Pannocchia et al. (2007), which is a combination of online and offline optimization. A table comprised of multiparametric solutions for multiple parameter values are created. In the online phase of the algorithm, an optimality check is performed. If none of the precomputed solutions satisfy the optimality conditions, an online step is followed to calculate the optimal solution is found. Ziogou et al. (2013) utilized multiparametric programming to speed-up the online solution time of nonlinear MPC. Specifically, an mpQP problem is formulated, based on which tighter bounds for the nonlinear program are constructed, which accelerate the computational performance.

Ferreau et al. (2008) demonstrated this concept for the solution of convex QPs in MPC. Based on their approach, it is assumed that the optimal control policy is found for an initial state vector. Given that 1 ) the current active set is known, 2) the optimal control actions are described by known piecewise affine functions with respect to the initial state vector, 3) a new initial state vector whose optimal solution is sought, and 4) guaranteeing primal and dual feasibility hold, the same KKT matrix is used to find the optimal solution for the new state vector dramatically reducing the online cost. The authors of the approach extended their findings and published an open source software package (Ferreau et al., 2014). The aforedescribed strategy was extended by Katz and Pistikopoulos (2020), where sampling is employed to uniformly build multiple critical regions that are used as a hot start for online MPC. An open question is whether such an approach can be extended to nonlinear systems, given that they have an increased complexity compared to convex QPs.

Multiparametric programming has also the potential to be utilized to solve dynamic optimization problems. To the knowledge of the authors, this topic has only been explored and published by Sakizlis (2003). Specifically, for problems described by a set of linear ordinary differential equations, it is demonstrated how the explicit continuous-time control laws can be obtained, as well as the switching points of the solution. The presented methodology was applied to relatively smallscale problems. Nevertheless, it can be used as the foundation for further developments in this field. Unlocking the capabilities of multiparametric programming in dynamic optimization is a substantially challenging task, however the benefits of having an explicit solution are remarkable and applicable in a wide range of problems in Process Systems Engineering.

\subsection{Multiparametric Distributed Model Predictive Control}

To minimize consumption of scarce resources and energy, integrated process systems with material recycling streams and energy integration are designed and operated in the chemical industry. Control of such systems using multiple single-loop proportional-integral-derivative (PID) controllers or MPC controllers by the conventional decentralized control system (DCS) architecture, will not capture the interactions between different control loops controlling the different subsystems of the process. The control system, hence, will not achieve the best possible closed-loop performance. On the other hand, a centralized control system, typically a single MPC controller capturing the interactions through a model, will not be able to respond within the time limit set by process dynamics and operating conditions when the number of controlled, manipulated, or process variables exceeds a particular threshold. The need to strike a balance between keeping the number of variables within acceptable limits like in DCS and capturing inter-loop interactions like in centralized MPC lead to the development of distributed model predictive control (DMPC) (Camponogara et al., 2002; Stewart et al., 2010). In this architecture, the DCS is improved to also pass on actuator settings of local controller(s) as inputs to controller(s) of other subsystems in the process. The MPC is the controller of choice as it takes advantage of a mathematical model. The MPC controllers in DMPC are not independent but interact with each other to optimize a centrally driven control objective (Christofides et al., 2013). Therefore, the model of a local MPC should not only be a function of the local manipulated variables but also a function of the variables that are being passed on as inputs from other controllers. This model can then be used to predict the local states over a finite time horizon into the future and calculate the optimal control move of the local controller by the conventional MPC control algorithm. 
A major challenge in DMPC is the prohibitive online computational costs of exchanging data and solving the optimal control problems of the multiple MPC controllers to converge to a solution. Another challenge is identifying the optimal distributed control structure based on either minimizing the number of inter-loop variables, improving robustness, or enhancing fault tolerant capabilities (Richards and How, 2007; Venkat et al., 2007; Jogwar, 2019). The optimal distributed control structure could also change during process operation as the conditions or operating modes change. Multiparametric programming helps shift the DMPC control and decomposition optimization problems offline, thereby keeping online computational loads manageable. The combined control algorithm will be referred to as "multiparametric distributed model predictive control" or mpDMPC.

While there are several studies reported in the literature on DMPC and on mpMPC, publications on mpDMPC are limited. Koehler and Borrelli (2013) formulated a one-step explicit DMPC for a building heating, ventilation and air conditioning (HVAC) system and compared the control performance against heuristic, "trim and respond" control. Kirubakaran et al. (2014) studied the feasibility, disturbance rejection capability, and robustness of cooperative mpDMPC for a benchmark quadruple tank problem. The authors reported improved state vector trajectory tracking, disturbance rejection, and robustness under cooperative mpDMPC compared to utilizing game theory DMPC.

The recent advancements in multiparametric programming algorithms reviewed in this paper and interest from the industry on energy-efficient integrated processes make mpDMPC a promising control strategy for applications to such systems and further research.

\section{CONCLUDING REMARKS}

In this contribution the background and recent developments in multiparametric programming have been presented. Advances focused in multiparametric programming theory, algorithms and applications have been discussed. In the former group of research efforts, particular attention has been paid on incorporating nonlinearity in the constraints of the problem, and solving the

\section{REFERENCES}

Åkesson, B. M., and Toivonen, H. T. (2006). A neural network model predictive controller. J. Process Contr. 16, 937-946. doi:10.1016/j.jprocont.2006.06.001

Acevedo, J., and Pistikopoulos, E. N. (1997). A multiparametric programming approach for linear process engineering problems under uncertainty. Ind. Eng. Chem. Res. 36, 717-728. doi:10.1021/ie9604511

Ahmadi-Moshkenani, P., Johansen, T. A., and Olaru, S. (2018). Combinatorial approach toward multiparametric quadratic programming based on characterizing adjacent critical regions. IEEE Trans. Automat. Contr. 63, 3221-3231. doi:10.1109/TAC.2018.2791479

Akbari, A., and Barton, P. I. (2018). An improved multi-parametric programming algorithm for flux balance analysis of metabolic networks. J. Opt. Theory Appl. 178, 502-537. doi:10.1007/s10957-018-1281-x resulting multiparametric nonlinear programming problem. As far as solution algorithms are concerned, both geometric-based and active set-based strategies have been proposed. Further developments on hybrid solution algorithms which balance the advantages of both approaches will be necessary to tackle largescale problems. In the latter group of research contributions, mpMPC remains the most studied application, motivated by its benefits in solving online optimization problems. However, it is highlighted through this paper that multiparametric programming is much broader than mpMPC, with exciting applications such as in solving multiscale, and multilevel optimization programs. Additionally, an outlook in future developments in the field of multiparametric programming has been included. The integration of data science and multiparametric programming, robust optimization and control, as well as complexity reduction techniques are still areas of development. It is expected that these fields, together with the aforedescribed recently published developments, will be at the heart of research within this fascinating world.

\section{AUTHOR CONTRIBUTIONS}

IP: Methodology, Formal Analysis, Data Collection, Writing original draft, DK: Data Collection, Formal Analysis, Writing review and editing, BB: Data Collection, Formal Analysis, Writing - review and editing, SA: Data Collection, Formal Analysis, Writing - review and editing, HG: Data Collection, Formal Analysis, Writing - review and editing, JK: Writing - review and editing, ND: Writing - review and editing, EP: Conceptualization, Supervision, Project administration, Funding acquisition.

\section{FUNDING}

Financial support from the National Science Foundation (Grant No. 1705423), Texas A\&M Energy Institute, the Clean Energy Smart Manufacturing Innovation Institute (CESMII), and the Rapid Advancement in Process Intensification Deployment (RAPID SYNOPSIS Project - DE-EE0007888-09-04) Institute is gratefully acknowledged.

Alessio, A., and Bemporad, A. (2009). “A survey on explicit model predictive control," in Nonlinear model predictive control. New York: Springer, 345-369.

Arce, A., Panos, C., Bordons, C., and Pistikopoulos, E. N. (2011). Design and experimental validation of an explicit mpc controller for regulating temperature in pem fuel cell systems. IFAC Proceedings Volumes 44, 2476-2481. doi:10. 3182/20110828-6-IT-1002.02819

Avraamidou, S., and Pistikopoulos, E. N. (2017a). "A multi-parametric bi-level optimization strategy for hierarchical model predictive control," in Computer aided chemical engineering. Amsterdam, Netherlands: Elsevier, 40, 1591-1596.

Avraamidou, S., and Pistikopoulos, E. N. (2017b). A multiparametric mixedinteger bi-level optimization strategy for supply chain planning under demand uncertainty. IFAC-PapersOnLine. 50, 10178-10183. doi:10.1016/j.ifacol.2017. 08.1766

Avraamidou, S., and Pistikopoulos, E. N. (2018). “A novel algorithm for the global solution of mixed-integer bi-level multi-follower problems and its application 
to planning scheduling integration," in 2018 European Control Conference (ECC),Limassol, Cyprus, June 12-15, 2018, (IEEE). doi:10.23919/ECC.2018. 8550351

Avraamidou, S., and Pistikopoulos, E. N. (2019a). B-pop: Bi-level parametric optimization toolbox. Comput. Chem. Eng. 122, 193-202. doi:10.1016/j. compchemeng.2018.07.007

Avraamidou, S., and Pistikopoulos, E. N. (2019b). "A global optimization algorithm for the solution of tri-level mixed-integer quadratic programming problems," in World Congress on Global Optimization. New York: Springer, 579-588.

Avraamidou, S., and Pistikopoulos, E. N. (2019c). Multi-parametric global optimization approach for tri-level mixed-integer linear optimization problems. J. Global Optim. 74, 443-465. doi:10.1007/s10898-018-0668-4

Avraamidou, S., and Pistikopoulos, E. N. (2019d). A multi-parametric optimization approach for bilevel mixed-integer linear and quadratic programming problems. Comput. Chem. Eng. 125, 98-113. doi:10.1016/j. compchemeng.2019.01.021

Avraamidou, S., and Pistikopoulos, E. N. (2020). Adjustable robust optimization through multi-parametric programming. Optimization Letters 14, 873-887. doi:10.1007/s11590-019-01438-5

Axehill, D., Besselmann, T., Raimondo, D. M., and Morari, M. (2014). A parametric branch and bound approach to suboptimal explicit hybrid mpc. Automatica. 50, 240-246. doi:10.1016/j.automatica.2013.10.004

Baotic, M. (2002). An algorithm for multiparametric quadratic programming. ETH Zurich, Technical Report.

Barnett, S. (1968). A simple class of parametric linear programming problems. Oper. Res. 16, 1160-1165. doi:10.1287/opre.16.6.1160

Bayat, F., Johansen, T. A., and Jalali, A. A. (2010). "Managing time-storage complexity in point location problem: Application to explicit model predictive control," in 18th Mediterranean Conference on Control and Automation, Marrakech, Morocco, June 23-25, 2010, (IEEE). doi:10.1109/ MED.2010.5547804

Bayer, F. A., Brunner, F. D., Lazar, M., Wijnand, M., and Allgöwer, F. (2016). “A tube-based approach to nonlinear explicit mpc," in 2016 IEEE 55th Conference on Decision and Control (CDC), Las Vegas, NV, December 12-14, 2016, (IEEE). doi:10.1109/CDC.2016.7798884

Bemporad, A. (2015). A multiparametric quadratic programming algorithm with polyhedral computations based on nonnegative least squares. IEEE Transactions on Automatatic Control. 60, 2892-2903. doi:10.1109/TAC.2015. 2417851

Bemporad, A., Borrelli, F., and Morari, M. (2003). Min-max control of constrained uncertain discrete-time linear systems. IEEE Trans. Automat. Contr. 48, 1600-1606. doi:10.1109/TAC.2003.816984

Bemporad, A., Borrelli, F., and Morari, M. (2002a). Model predictive control based on linear programming the explicit solution. IEEE Trans. Automat. Contr. 47, 1974-1985. doi:10.1109/TAC.2002.805688

Bemporad, A., Morari, M., Dua, V., and Pistikopoulos, E. (2002b). The explicit linear quadratic regulator for constrained systems. Automatica. 38, 3-20. doi:10.1016/S0005-1098(01)00174-1

Bemporad, A., and Filippi, C. (2006). An algorithm for approximate multiparametric convex programming. Comput. Optim. Appl. 35, 87-108. doi:10.1109/LCSYS.2019.2922639

Bemporad, A., and Morari, M. (1999). Robust model predictive control: A survey," in Robustness in identification and control. New York: Springer. 207-226.

Ben-Tal, A., Goryashko, A., Guslitzer, E., and Nemirovski, A. (2004). Adjustable robust solutions of uncertain linear programs. Math. Program. 99, 351-376. doi:10.1007/s10107-003-0454-y

Benson, H. P. (1982). Algorithms for parametric nonconvex programming. J. Optim. Theor. Appl. 38, 319-340.

Burnak, B., Diangelakis, N. A., Katz, J., and Pistikopoulos, E. N. (2019a). Integrated process design, scheduling, and control using multiparametric programming. Comput. Chem. Eng. 125, 164-184. doi:10.1016/j.compchemeng.2019.03.004

Burnak, B., Diangelakis, N. A., and Pistikopoulos, E. N. (2019b). Towards the grand unification of process design, scheduling, and control-utopia or reality? Processes 7, 7070461. doi:10.3390/pr7070461

Burnak, B., Diangelakis, N. A., and Pistikopoulos, E. N. (2020a). Integrated Process Design and Operational Optimization via Multiparametric Programming. Morgan \& Claypool Publishers.
Burnak, B., Katz, J., and Pistikopoulos, E. N. (2020b). A space exploration algorithm for multiparametric programming via delaunay triangulation. Optim. Eng. [Epub ahead of print]. doi:10.1007/s11081-020-09535-6

Burnak, B., Katz, J., Diangelakis, N. A., and Pistikopoulos, E. N. (2018), Simultaneous process scheduling and control: A multiparametric programming-based approach. Ind. Eng. Chem. Res. 57, 3963-3976. doi:10. 1021/acs.iecr.7b04457

Burnak, B., and Pistikopoulos, E. N. (2020). Integrated process design, scheduling, and model predictive control of batch processes with closed-loop implementation. AIChE J. 66, e16981. doi:10.1002/aic.16981

Camponogara, E., Jia, D., Krogh, B. H., and Talukdar, S. (2002). Distributed model predictive control. IEEE control systems magazine. 22, 44-52. doi:10.1109/ACC. 2001.946306

Caspari, A., Tsay, C., Mhamdi, A., Baldea, M., and Mitsos, A. (2020). The integration of scheduling and control: Top-down vs. bottom-up. J. Process Contr. 91, 50-62. doi:10.1016/j.jprocont.2020.05.008

Charitopoulos, V., and Dua, V. (2016). Explicit model predictive control of hybrid systems and multiparametric mixed integer polynomial programming. AIChE J. 62, 3441-3460. doi:10.1002/aic.15396

Charitopoulos, V. M., Papageorgiou, L. G., and Dua, V. (2017). Multi-parametric linear programming under global uncertainty. AIChE J. 63, 3871-3895. doi:10. 1002/aic.15755

Charitopoulos, V. M., Papageorgiou, L. G., and Dua, V. (2018). Multi-parametric mixed integer linear programming under global uncertainty. Comput. Chem. Eng. 116, 279-295. doi:10.1016/j.compchemeng.2018.04.015

Charitopoulos, V. M., Papageorgiou, L. G., and Dua, V. (2019). Closed-loop integration of planning, scheduling and multi-parametric nonlinear control. Comput. Chem. Eng. 122, 172-192. doi:10.1016/j.compchemeng. 2018.06.021

Chen, S., Saulnier, K., Atanasov, N., Lee, D. D., Kumar, V., and Pappas, G. J., et al. (2018). "Approximating explicit model predictive control using constrained neural networks," in 2018 Annual American control conference (ACC), Milwaukee, WI, June 27-29, 2018, (IEEE). doi:10.23919/ACC.2018.8431275

Christofides, P. D., Scattolini, R., de la Pena, D. M., and Liu, J. (2013). Distributed model predictive control: A tutorial review and future research directions. Comput. Chem. Eng. 51, 21-41. doi:10.1016/j.compchemeng.2012.05.011

Dempe, S. (2002). Foundations of bilevel programming. Springer Science \& Business Media.

Di Cairano, S., Yanakiev, D., Bemporad, A., Kolmanovsky, I. V., and Hrovat, D. (2008). "An mpc design flow for automotive control and applications to idle speed regulation," in 2008 47th IEEE Conference on Decision and Control, Cancun, Mexico, December 9-11, 2008, (IEEE). doi:10.1109/CDC.2008.4738865

Diangelakis, N. A., Burnak, B., Katz, J., and Pistikopoulos, E. N. (2017). Process design and control optimization: A simultaneous approach by multi-parametric programming. AIChE J. 63, 4827-4846. doi:10.1002/aic.15825

Diangelakis, N. A., Pappas, I. S., and Pistikopoulos, E. N. (2018). On multiparametric/explicit nmpc for quadratically constrained problems. IFAC-PapersOnline 20, 490-495. doi:10.1016/j.ifacol.2018.11.066

Domínguez, L. F., and Pistikopoulos, E. N. (2010). Multiparametric programming based algorithms for pure integer and mixed-integer bilevel programming problems. Comput. Chem. Eng. 34, 2097-2106. doi:10.1016/j.compchemeng. 2010.07.032

Domínguez, L. F., and Pistikopoulos, E. N. (2013). A quadratic approximationbased algorithm for the solution of multiparametric mixed-integer nonlinear programming problems. AIChE J. 59, 483-495. doi:10.1002/aic.13838

Drgoňa, J., Klaučo, M., Janeček, F., and Kvasnica, M. (2017). Optimal control of a laboratory binary distillation column via regionless explicit mpc. Comput. Chem. Eng. 96, 139-148. doi:10.1016/j.compchemeng.2016.10.003

Dua, P. (2005). Model based and parametric control for drug delivery systems. Ph.D. thesis, London: University of London.

Dua, P., Kouramas, K., Dua, V., and Pistikopoulos, E. N. (2008). Mpc on a chiprecent advances on the application of multi-parametric model-based control. Comput. Chem. Eng. 32, 754-765. doi:10.1016/j.compchemeng.2007.03.008

Dua, V. (2015). Mixed integer polynomial programming. Comput. Chem. Eng. 72, 387-394. doi:10.1016/j.compchemeng.2014.07.020

Dua, V., Bozinis, N. A., and Pistikopoulos, E. N. (2002). A multiparametric programming approach for mixed-integer quadratic engineering problems. Comput. Chem. Eng. 26, 715-733. doi:10.1016/S0098-1354(01)00797-9 
Dua, V., and Pistikopoulos, E. N. (1999). Algorithms for the solution of multiparametric mixed-integer nonlinear optimization problems. Ind. Eng. Chem. Res. 38, 3976-3987. doi:10.1023/A:1019241000636

Dua, V., and Pistikopoulos, E. N. (2000). An algorithm for the solution of multiparametric mixed integer linear programming problems. Ann. Oper. Res. 99, 123-139. doi:10.1023/A:1019241000636

Efron, B., Hastie, T., Johnstone, I., Tibshirani, R., et al. (2004). Least angle regression. Ann. Stat. 32, 407-499. doi:10.1214/009053604000000067

Emekli, M. E., and Güvenç, B. A. (2016). Explicit mimo model predictive boost pressure control of a two-stage turbocharged diesel engine. IEEE transactions on control systems technology. 25, 521-534. doi:10.1109/TCST.2016.2554558

Ettefagh, M. H., Naraghi, M., and Towhidkhah, F. (2019). Position control of a flexible joint via explicit model predictive control: An experimental implementation. Emerging Science Journal. 3, 146-156. doi:10.28991/esj2019-01177

FaÃsca, N. P., Rustem, B., and Dua, V. (2011). Bilevel and Multilevel Programming. New Jersey: John Wiley \& Sons, Ltd.

Faísca, N. P., Dua, V., Rustem, B., Saraiva, P. M., and Pistikopoulos, E. N. (2007). Parametric global optimisation for bilevel programming. J. Global Optim. 38, 609-623. doi:10.1007/s10898-006-9100-6

Faísca, N. P., Kosmidis, V. D., Rustem, B., and Pistikopoulos, E. N. (2009). Global optimization of multi-parametric milp problems. J. Global Optim. 45, 131-151. doi:10.1007/s10898-008-9398-3

Fang, J., Huang, C., Tang, T., and Wang, Z. (2020). Parallel programming models for heterogeneous many-cores : A survey. arXiv. 2005.04094, 1-30.

Feller, C., Johansen, T. A., and Olaru, S. (2013). An improved algorithm for combinatorial multi-parametric quadratic programming. Automatica. 49, 1370-1376. doi:10.1016/j.automatica.2013.02.022

Ferreau, H. J., Bock, H. G., and Diehl, M. (2008). An online active set strategy to overcome the limitations of explicit mpc. Int. J. Robust Nonlinear Control: IFAC-Affiliated Journal. 18, 816-830. doi:10.1002/rnc.1251

Ferreau, H. J., Kirches, C., Potschka, A., Bock, H. G., and Diehl, M. (2014). qpoases: A parametric active-set algorithm for quadratic programming. Mathematical Programming Computation. 6, 327-363. doi:10.1007/s12532-014-0071-1

Fiacco, A. V. (1976). Sensitivity analysis for nonlinear programming using penalty methods. Math. Program. 10, 287-311.

Fiacco, A. V. (1983). Introduction to sensitivity analysis and stability analysis in nonlinear programming. Amsterdam, Netherlands: Elsevier.

Fiacco, A. V., and Kyparisis, J. (1986). Convexity and concavity properties of the optimal value function in parametric nonlinear programming. J. Optim. Theor. Appl. 48, 95-126.

Filippi, C. (2004). An algorithm for approximate multiparametric linear programming. J. Optim. Theor. Appl. 120, 73-95. doi:10.1023/B:JOTA. 0000012733.44020.54

Fotiou, I., Rostalski, P., Parrilo, P., and Morari, M. (2006). Parametric optimization and optimal control using algebraic geometry methods. Int. J. Contr. 79, 1340-1358. doi:10.1080/00207170600726592

Gal, T. (1975). Rim multiparametric linear programming. Manag. Sci. 21, 567-575.

Gal, T., and Nedoma, J. (1972). Multiparametric linear programming. Manag. Sci. $18,406-422$.

Grancharova, A., Johansen, T. A., and Kocijan, J. (2004). Explicit model predictive control of gas-liquid separation plant via orthogonal search tree partitioning. Comput. Chem. Eng. 28, 2481-2491. doi:10.1016/j.compchemeng.2004.06.010

Grimstad, B., and Andersson, H. (2019). Relu networks as surrogate models in mixed-integer linear programs. Comput. Chem. Eng. 131, 106580. doi:10.1016/j. compchemeng.2019.106580

Gupta, A., Bhartiya, S., and Nataraj, P. (2011). A novel approach to multiparametric quadratic programming. Automatica. 47, 2112-2117. doi:10.1016/j.automatica.2011.06.019

Hale, E. T. (2005). Numerical methods for d-parametric nonlinear programming with chemical process control and optimization applications. Ph.D. thesis. University of Texas at Austin.

Hale, E. T., and Qin, S. J. (2004). Multi-parametric nonlinear programming and the evaluation of implicit optimization model adequacy. IFAC Proceedings Volumes. 37, 449-454. doi:10.1016/S1474-6670(17)31850-5

Hanin, B., and Sellke, M. (2018). Approximating continuous functions by relu nets of minimal width. arXiv. 1710.11278, 1-13.
Hastie, T., Rosset, S., Tibshirani, R., and Zhu, J. (2004). The entire regularization path for the support vector machine. J. Mach. Learn. Res. 5, 1391-1415. doi:10. 5555/1005332.1044706

Herceg, M., Jones, C. N., Kvasnica, M., and Morari, M. (2015). Enumeration-based approach to solving parametric linear complementarity problems. Automatica. 62, 243-248. doi:10.1016/j.automatica.2015.09.019

Herceg, M., Kvasnica, M., Jones, C. N., and Morari, M. (2013). "Multi-parametric toolbox 3.0," in 2013 European control conference (ECC), Zurich, Switzerland, July 17-19, 2013, (IEEE). doi:10.23919/ECC.2013.6669862

Jia, C., Wang, X., Zhou, K., and Xianqing, D. (2019). "Sensorless explicit model predictive control for ipmsm drives," in 2019 IEEE 2nd International Conference on Automation, Electronics and Electrical Engineering, Shenyang, China, November 22-24, 2019, (IEEE). doi:10.1109/ AUTEEE48671.2019.9033424

Jia, Z., and Ierapetritou, M. G. (2006). Uncertainty analysis on the righthand side for milp problems. AIChE J. 52, 2486-2495. doi:10.1002/aic.10842

Jiang, Y., Oravec, J., Houska, B., and Kvasnica, M. (2020). Parallel mpc for linear systems with input constraints. IEEE Trans. Automat. Contr. doi:10.1109/TAC. 2020.3020827

Jogwar, S. S. (2019). Distributed control architecture synthesis for integrated process networks through maximization of strength of input-output impact. J. Process Contr. 83, 77-87. doi:10.1016/j.jprocont.2019.08.009

Johansen, T. A. (2004). Approximate explicit receding horizon control of constrained nonlinear systems. Automatica. 40, 293-300. doi:10.1016/j. automatica.2003.09.021

Johansen, T. A. (2002). "On multi-parametric nonlinear programming and explicit nonlinear model predictive control," in IEEE Conference on Decision and Control, Las Vegas, NV, December 10-13, 2002, (IEEE). doi:10.1109/CDC. 2002.1184260

Jones, C. N., Kerrigan, E. C., and Maciejowski, J. M. (2007). Lexicographic perturbation for multiparametric linear programming with applications to control. Automatica. 43, 1808-1816. doi:10.1016/j.automatica.2007.03.008

Karasuyama, M., Harada, N., Sugiyama, M., and Takeuchi, I. (2012). Multiparametric solution-path algorithm for instance-weighted support vector machines. Mach. Learn. 88, 297-330. doi:10.1109/MLSP.2011.6064551

Karg, B., and Lucia, S. (2020). Efficient representation and approximation of model predictive control laws via deep learning. IEEE Trans. Cyber. 50 (9), 3866-3878.

Kassa, A. M., and Kassa, S. M. (2013). A multi-parametric programming algorithm for special classes of non-convex multilevel optimization problems. An Int. J. Optim. Control Theor. Appl. (IJOCTA). 3, 133-144. doi:10.11121/ijocta.01.2013.00156

Kassa, A. M., and Kassa, S. M. (2016). A branch-and-bound multi-parametric programming approach for non-convex multilevel optimization with polyhedral constraints. J. Global Optim. 64, 745-764. doi:10.1007/s10898015-0341-0

Kassa, A. M., and Kassa, S. M. (2017). Deterministic solution approach for some classes of nonlinear multilevel programs with multiple followers. J. Global Optim. 68, 729-747. doi:10.1007/s10898-017-0502-4

Katz, J., Pappas, I., Avraamidou, S., and Pistikopoulos, E. (2020a). "The integration of explicit mpc and relu based neural networks," in 21st IFAC World Congress.

Katz, J., Pappas, I., Avraamidou, S., and Pistikopoulos, E. N. (2020b). Integrating deep learning models and multiparametric programming. Comput. Chem. Eng. 136, 106801. doi:10.1016/j.compchemeng.2020.106801

Katz, J., and Pistikopoulos, E. N. (2020). A partial multiparametric optimization strategy to improve the computational performance of model predictive control. Comput. Chem. Eng. 142, 107057. doi:10.1016/j.compchemeng.2020.107057

Kirubakaran, V., Radhakrishnan, T., and Sivakumaran, N. (2014). Distributed multiparametric model predictive control design for a quadruple tank process. Measurement. 47, 841-854. doi:10.1016/j.measurement.2013.10.011

Klaučo, M., Kalúz, M., and Kvasnica, M. (2017). Real-time implementation of an explicit mpc-based reference governor for control of a magnetic levitation system. Contr. Eng. Pract. 60, 99-105. doi:10.1016/j.conengprac.2017.01.001

Koehler, S., and Borrelli, F. (2013). "Building temperature distributed control via explicit mpc and "trim and respond" methods," in 2013 European Control Conference (ECC), Zurich, Switzerland, July 17-19, 2013, (IEEE). doi:10.23919/ ECC.2013.6669781

Kojima, M. (1979). A complementary pivoting approach to parametric nonlinear programming. Math. Oper. Res. 4, 464-477. doi:10.1287/moor.4.4.464 
Kojima, M., and Hirabayashi, R. (1984). "Continuous deformation of nonlinear programs," in Sensitivity, Stability and Parametric Analysis. New York: Springer, 150-198.

Kothare, M. V., Balakrishnan, V., and Morari, M. (1996). Robust constrained model predictive control using linear matrix inequalities. Automatica. 32, 1361-1379. doi:10.1016/0005-1098(96)00063-5

Kouramas, K. I., Panos, C., Faísca, N. P., and Pistikopoulos, E. N. (2013). An algorithm for robust explicit/multi-parametric model predictive control. Automatica. 49, 381-389. doi:10.1016/j.automatica.2012.11.035

Kuhn, D., Wiesemann, W., and Georghiou, A. (2011). Primal and dual linear decision rules in stochastic and robust optimization. Math. Program. 130, 177-209. doi:10.1007/s10107-009-0331-4

Kvasnica, M., Takács, B., Holaza, J., and Di Cairano, S. (2015). On region-free explicit model predictive control. in 2015 54th IEEE Conference on Decision and Control (CDC), Osaka, Japan, December 15-18, 2015, (IEEE). doi:10.1109/ CDC.2015.7402788

Langson, W., Chryssochoos, I., Raković, S., and Mayne, D. Q. (2004). Robust model predictive control using tubes. Automatica. 40, 125-133. doi:10.1016/j. automatica.2003.08.009

Lasheen, A., Saad, M. S., Emara, H. M., and Elshafei, A. L. (2019). Tube-based explicit model predictive output-feedback controller for collective pitching of wind turbines. Renew. Energy 131, 549-562. doi:10.1016/j.renene.2018.07.033

Lee, J., and Chang, H.-J. (2019). Multi-parametric model predictive control for autonomous steering using an electric power steering system. Proc. Inst. Mech. Eng. - Part D J. Automob. Eng. 233, 3391-3402. doi:10.1177/0954407018824773

Li, Z., and Ierapetritou, M. G. (2007). A new methodology for the general multiparametric mixed-integer linear programming (milp) problems. Ind. Eng. Chem. Res. 46, 5141-5151. doi:10.1021/ie070148s

Li, Z., and Ierapetritou, M. G. (2010). A method for solving the general parametric linear complementarity problem. Ann. Oper. Res. 181, 485-501. doi:10.1007/ s10479-010-0770-6

Linder, A., and Kennel, R. (2005). "Model predictive control for electrical drives," in 2005 IEEE 36th Power Electronics Specialists Conference, Recife, Brazil, June 16, 2005, (IEEE). doi:10.1109/PESC.2005.1581874

Lovelett, R. J., Dietrich, F., Lee, S., and Kevrekidis, I. G. (2020). Some manifold learning considerations toward explicit model predictive control. AIChE J. 66, e16881. doi:10.1002/aic.16881

Mandler, J. A., Bozinis, N. A., Sakizlis, V., Pistikopoulos, E. N., Prentice, A. L., Ratna, H., et al. (2006). Parametric model predictive control of air separation. IFAC Proceedings Volumes 39, 617-622. doi:10.3182/20060402-4-BR-2902. 00617

Mariéthoz, S., and Morari, M. (2008). Explicit model-predictive control of a pwm inverter with an lcl filter. IEEE Trans. Ind. Electron. 56, 389-399. doi:10.1109/ TIE.2008.2008793

Mayne, D. Q. (2014). Model predictive control: recent developments and future promise. Automatica. 50, 2967-2986. doi:10.1016/j.automatica.2014.10.128

Mayne, D. Q., Seron, M. M., and Raković, S. (2005). Robust model predictive control of constrained linear systems with bounded disturbances. Automatica. 41, 219-224. doi:10.1016/j.automatica.2004.08.019

McInerney, I., Constantinides, G. A., and Kerrigan, E. C. (2018). A survey of the implementation of linear model predictive control on fpgas. IFAC. 51, 381-387. doi:10.1016/j.ifacol.2018.11.063

Mitsos, A., and Barton, P. I. (2009). Parametric mixed-integer 0-1 linear programming: the general case for a single parameter. Eur. J. Oper. Res. 194, 663-686. doi:10.1016/j.ejor.2008.01.007

Mohideen, M., Perkins, J., and Pistikopoulos, E. (1996). Optimal synthesis and design of dynamic systems under uncertainty. Comput. Chem. Eng. 20, S895-S900. doi:10.1016/0098-1354(96)00157-3

Mohideen, M., Perkins, J., and Pistikopoulos, E. (1997). Robust stability considerations in optimal design of dynamic systems under uncertainty. J. Process Contr. 7, 371-385. doi:10.1016/S0959-1524(97)00014-0

Narciso, D. A. C. (2009). Developments in nonlinear multiparametric programming and control. Ph.D. thesis, London: Imperial College London.

Naus, G., Van Den Bleek, R., Ploeg, J., Scheepers, B., van de Molengraft, R., and Steinbuch, M. (2008). "Explicit mpc design and performance evaluation of an acc stop-\&-go," in 2008 American Control Conference, Seattle, WA, June 11-13, 2018, (IEEE), 224-229. doi:10.1109/ACC.2008.4586495
Oberdieck, R., Diangelakis, N. A., Nascu, I., Papathanasiou, M. M., Sun, M., Avraamidou, S., et al. (2016a). On multi-parametric programming and its applications in process systems engineering. Chem. Eng. Res. Des. 116, 61-82. doi:10.1016/j.cherd.2016.09.034

Oberdieck, R., Diangelakis, N. A., Papathanasiou, M. M., Nascu, I., and Pistikopoulos, E. N. (2016b). Pop-parametric optimization toolbox. Ind. Eng. Chem. Res. 55, 8979-8991. doi:10.1021/acs.iecr.6b01913

Oberdieck, R., Diangelakis, N. A., Avraamidou, S., and Pistikopoulos, E. N. (2017a). On unbounded and binary parameters in multi-parametric programming: applications to mixed-integer bilevel optimization and duality theory. J. Global Optim. 69, 587-606. doi:10.1007/s10898-016-0463-z

Oberdieck, R., Diangelakis, N. A., and Pistikopoulos, E. N. (2017b). Explicit model predictive control: A connected-graph approach. Automatica. 76, 103-112. doi:10.1016/j.automatica.2016.10.005

Oberdieck, R., and Pistikopoulos, E. N. (2015). Explicit hybrid model-predictive control: the exact solution. Automatica. 58, 152-159. doi:10.1016/j.automatica. 2015.05.021

Oberdieck, R., and Pistikopoulos, E. N. (2016). "Parallel computing in multiparametric programming," in Computer aided chemical engineering. Amsterdam, Netherlands: Elsevier, 38. 169-174.

Oberdieck, R., Wittmann-Hohlbein, M., and Pistikopoulos, E. N. (2014). A branch and bound method for the solution of multiparametric mixed integer linear programming problems. J. Global Optim. 59, 527-543. doi:10.1007/s10898-014-0143-9

Ogumerem, G. S., and Pistikopoulos, E. N. (2019). Parametric optimization and control toward the design of a smart metal hydride refueling system. AIChE J. 65, e16680. doi:10.1002/aic.16680

Ogumerem, G. S., and Pistikopoulos, E. N. (2020). Parametric optimization and control for a smart proton exchange membrane water electrolysis (pemwe) system. J. Process Contr. 91, 37-49. doi:10.1016/j.jprocont.2020.05.002

Onel, M., Burnak, B., and Pistikopoulos, E. N. (2019). Integrated data-driven process monitoring and explicit fault-tolerant multiparametric control. Ind. Eng. Chem. Res. 59, 2291-2306. doi:10.1021/acs.iecr.9b04226

Pannocchia, G., Rawlings, J. B., and Wright, S. J. (2007). Fast, large-scale model predictive control by partial enumeration. Automatica. 43, 852-860. doi:10. 1016/j.automatica.2006.10.019

Pappas, I., Diangelakis, N. A., and Pistikopoulos, E. N. (2020a). The exact solution of multiparametric quadratically constrained quadratic programming problems. J. Global Optim. doi:10.1007/s10898-020-00933-9

Pappas, I., Diangelakis, N. A., and Pistikopoulos, E. N. (2020b). "Explicit nonlinear model predictive control for the operation of a cstr near an unstable steadystate," in IFAC World Congress 2020 (IFAC).

Parisini, T., and Zoppoli, R. (1995). A receding-horizon regulator for nonlinear systems and a neural approximation. Automatica. 31, 1443-1451. doi:10.1016/ 0005-1098(95)00044-W

Patrinos, P., and Sarimveis, H. (2010). A new algorithm for solving convex parametric quadratic programs based on graphical derivatives of solution mappings. Automatica. 46, 1405-1418. doi:10.1016/j.automatica.2010. 06.008

Pertsinidis, A., Grossmann, I. E., and McRae, G. J. (1998). Parametric optimization of milp programs and a framework for the parametric optimization of minlps. Comput. Chem. Eng. 22, S205-S212. doi:10.1016/S0098-1354(98)00056-8

Petsagkourakis, P., and Theodoropoulos, C. (2018). "Data driven reduced order nonlinear multiparametric mpc for large scale systems," in Computer Aided Chemical Engineering. Amsterdam, Netherlands: Elsevier, 43. 1249-1254.

Pistikopoulos, E. N. (2012). From multi-parametric programming theory to mpcon-a-chip multi-scale systems applications. Comput. Chem. Eng. 47, 57-66. doi:10.1016/j.compchemeng.2012.06.031

Pistikopoulos, E. N., and Diangelakis, N. A. (2016). Towards the integration of process design, control and scheduling: Are we getting closer? Comput. Chem. Eng. 91, 85-92. doi:10.1016/j.compchemeng.2015.11.002

Pistikopoulos, E. N., Diangelakis, N. A., Oberdieck, R., Papathanasiou, M. M., Nascu, I., and Sun, M. (2015). Paroc-an integrated framework and software platform for the optimisation and advanced model-based control of process systems. Chem. Eng. Sci. 136, 115-138. doi:10.1016/j.ces.2015.02.030

Pistikopoulos, E. N., Dua, V., Bozinis, N. A., Bemporad, A., and Morari, M. (2000). On-line optimization via off-line parametric optimization tools. Comput. Chem. Eng. 24, 183-188. doi:10.1016/S0098-1354(00)00510-X 
Pistikopoulos, E. N. (2009). Perspectives in multiparametric programming and explicit model predictive control. AIChE journal. 55, 1918-1925. doi:10.1002/ aic. 11965

Pramanik, S., and Roy, T. K. (2007). Fuzzy goal programming approach to multilevel programming problems. Eur. J. Oper. Res. 176, 1151-1166. doi:10. 1016/j.ejor.2005.08.024

Qin, S. J., and Badgwell, T. A. (2000). "An overview of nonlinear model predictive control applications," in Nonlinear model predictive control. New York: Springer. 369-392.

Qin, S. J., and Badgwell, T. A. (2003). A survey of industrial model predictive control technology. Contr. Eng. Pract. 11, 733-764. doi:10.1016/S09670661(02)00186-7

Rafiei, M., and Ricardez-Sandoval, L. A. (2020). New frontiers, challenges, and opportunities in integration of design and control for enterprise-wide sustainability. Comput. Chem. Eng. 132, 106610. doi:10.1016/j. compchemeng.2019.106610

Richards, A., and How, J. P. (2007). Robust distributed model predictive control. Int. J. Contr. 80, 1517-1531. doi:10.1080/00207170701491070

Ryu, J.-H., Dua, V., and Pistikopoulos, E. N. (2004). A bilevel programming framework for enterprise-wide process networks under uncertainty. Comput. Chem. Eng. 28, 1121-1129. doi:10.1016/j.compchemeng.2003.09.021

Sakawa, M., and Matsui, T. (2014). Interactive fuzzy stochastic multi-level 0-1 programming using tabu search and probability maximization. Expert systems with applications. 41, 2957-2963. doi:10.1016/j.eswa.2013.10.027

Sakizlis, V. (2003). Design of of model-based controllers via parametric programming. Ph.D. Thesis. London: Imperial College London.

Sakizlis, V., Kakalis, N. M., Dua, V., Perkins, J. D., and Pistikopoulos, E. N. (2004). Design of robust model-based controllers via parametric programming. Automatica. 40, 189-201. doi:10.1016/j.automatica.2003.08.011

Sakizlis, V., Perkins, J., and Pistikopoulos, E. (2003). Parametric controllers in simultaneous process and control design optimization. Ind. Eng. Chem. Res. 42, 4545-4563. doi:10.1016/S1570-7946(03)80442-X

Scaglioni, B., Previtera, L., Martin, J., Norton, J., Obstein, K. L., and Valdastri, P. (2019). Explicit model predictive control of a magnetic flexible endoscope. IEEE Robot Autom Lett. 4, 716-723. doi:10.1109/LRA.2019.2893418

Schechter, M. (1987). Polyhedral functions and multiparametric linear programming. J. Optim. Theor. Appl. 53, 269-280.

Shokry, A., Dombayci, C., and Espuña, A. (2016). "Multiparametric metamodels for model predictive control of chemical processes," in Computer Aided Chemical Engineering. Amsterdam, Netherlands: Elsevier, 38. 937-942.

Shokry, A., Medina-González, S., and Espuña, A. (2017). "Mixed-integer multiparametric approach based on machine learning techniques," in Computer Aided Chemical Engineering. Amsterdam, Netherlands: Elsevier, 40. $451-456$

Spjøtvold, J., Kerrigan, E. C., Jones, C. N., TøNdel, P., and Johansen, T. A. (2006). On the facet-to-facet property of solutions to convex parametric quadratic programs. Automatica. 42, 2209-2214. doi:10.1016/j.automatica.2006.06.026

Stewart, B. T., Venkat, A. N., Rawlings, J. B., Wright, S. J., and Pannocchia, G. (2010). Cooperative distributed model predictive control. Syst. Contr. Lett. 59, 460-469. doi:10.1016/j.sysconle.2010.06.005

Tøndel, P., and Johansen, T. A. (2002). Complexity reduction in explicit linear model predictive control. IFAC Proceedings Volumes 35, 189-194. doi:10.3182/ 20020721-6-ES-1901.00600

Tøndel, P., Johansen, T. A., and Bemporad, A. (2003). An algorithm for multiparametric quadratic programming and explicit mpc solutions. Automatica. 39, 489-497. doi:10.1016/S0005-1098(02)00250-9

Takács, B., Števek, J., Valo, R., and Kvasnica, M. (2016). "Python code generation for explicit mpc in mpt," in 2016 European Control Conference (ECC), Aalborg, Denmark, June 29-July 1, (IEEE). doi:10.1109/ECC.2016.7810473

Tavernini, D., Metzler, M., Gruber, P., and Sorniotti, A. (2018). Explicit nonlinear model predictive control for electric vehicle traction control. IEEE Trans. Contr. Syst. Technol. 27, 1438-1451. doi:10.1109/TCST.2018.2837097
Tavernini, D., Vacca, F., Metzler, M., Savitski, D., Ivanov, V., Gruber, P., et al. (2019). An explicit nonlinear model predictive abs controller for electrohydraulic braking systems. IEEE Trans. Ind. Electron. 67, 3990-4001. doi:10. 1109/TIE.2019.2916387

Tejeda-Iglesias, M., Lappas, N. H., Gounaris, C. E., and Ricardez-Sandoval, L. (2019). Explicit model predictive controller under uncertainty: An adjustable robust optimization approach. J. Process Contr. 84, 115-132. doi:10.1016/j. jprocont.2019.09.002

Theunissen, J., Sorniotti, A., Gruber, P., Fallah, S., Ricco, M., Kvasnica, M., et al. (2019). Regionless explicit model predictive control of active suspension systems with preview. IEEE Trans. Ind. Electron. 67, 4877-4888. doi:10. 1109/TIE.2019.2926056

Tian, Y., Pappas, I., Burnak, B., Katz, J., and Pistikopoulos, E. N. (2020). Simultaneous design \& control of a reactive distillation system-a parametric optimization \& control approach. Chem. Eng. Sci. 230, 116232. doi:10.1016/j. ces.2020.116232

Tso, W. W., Burnak, B., and Pistikopoulos, E. N. (2020). Hy-pop: hyperparameter optimization of machine learning models through parametric programming. Comput. Chem. Eng. 139, 106902. doi:10.1016/j. compchemeng.2020.106902

Vadamalu, R. S., and Beidl, C. (2016). "Explicit mpc phev energy management using Markov chain based predictor: development and validation at engine-inthe-loop testbed," in 2016 European Control Conference (ECC), Aalborg, Denmark, June 29-July 1, 2016, (IEEE).

Venkat, A. N., Rawlings, J. B., and Wright, S. J. (2007). "Distributed model predictive control of large-scale systems," in Assessment and Future Directions of Nonlinear Model Predictive Control. New York: Springer. 591-605.

Wittmann-Hohlbein, M., and Pistikopoulos, E. N. (2013). On the global solution of multi-parametric mixed integer linear programming problems. J. Global Optim. 57, 51-73. doi:10.1016/j.compchemeng.2018.04.015

Wittmann-Hohlbein, M., and Pistikopoulos, E. N. (2014). Approximate solution of mp-milp problems using piecewise affine relaxation of bilinear terms. Comput. Chem. Eng. 61, 136-155. doi:10.1016/j.compchemeng.2013.10.009

Yuf, P., and Zeleny, M. (1976). Linear multiparametric programming by multicriteria simplex method. Manag. Sci. 23, 159-170.

Zhou, Y., and Spanos, C. J. (2016). "On a class of multi-parametric quadratic programming and its applications to machine learning," in 2016 IEEE 55th Conference on Decision and Control (CDC), Las Vegas, NV, December 12-14, 2016 (IEEE).

Zhuge, J., and Ierapetritou, M. G. (2014). Integration of scheduling and control for batch processes using multi-parametric model predictive control. AIChE J. 60, 3169-3183. doi:10.1002/aic.14509

Ziogou, C., Pistikopoulos, E. N., Georgiadis, M. C., Voutetakis, S., and Papadopoulou, S. (2013). Empowering the performance of advanced nmpc by multiparametric programming an application to a pem fuel cell system. Ind. Eng. Chem. Res. 52, 4863-4873. doi:10.1021/ie303477h

Ziogou, C., Voutetakis, S., Georgiadis, M. C., and Papadopoulou, S. (2018). Model predictive control $(\mathrm{mpc})$ strategies for pem fuel cell systems-a comparative experimental demonstration. Chem. Eng. Res. Des. 131, 656-670. doi:10.1016/j. cherd.2018.01.024

Conflict of Interest: The authors declare that the research was conducted in the absence of any commercial or financial relationships that could be construed as a potential conflict of interest.

Copyright (c) 2021 Pappas, Kenefake, Burnak, Avraamidou, Ganesh, Katz, Diangelakis and Pistikopoulos. This is an open-access article distributed under the terms of the Creative Commons Attribution License (CC BY). The use, distribution or reproduction in other forums is permitted, provided the original author(s) and the copyright owner(s) are credited and that the original publication in this journal is cited, in accordance with accepted academic practice. No use, distribution or reproduction is permitted which does not comply with these terms. 